\title{
Vertical changes during Begg's and PEA-A Comparative Study
}

\author{
Dr. Suchita Tarvade/Daokar ${ }^{1}$, Dr. Charushila Chaudhari ${ }^{2}$, \\ Dr. Handa Amit Satish ${ }^{3}$ \\ ${ }^{1,2,3}$ CSMSS Dental College \& Hospital, Aurangabad, Maharashtra, India
}

\begin{abstract}
The purpose of this study was to compare the vertical dimensional changes in bialveolar dental protrusion patients undergoing extraction of all four first premolars between the preadjusted edgewise appliance(PEA)(MBT) and the Begg appliance. The cephalometric records of 40 patients with Class I bimaxillary protrusion were selected and devided in two groups i.e Begg or PEA mechanotherapy. The age group of 18-25 years was selected for both groups. Skeletal and dental changes were analysed in both groups on lateral cephalograms taken pre (T1)\& post (T2) treatment. A student t-test was used to analyse the treatment changes.

Significant increase in face height and extrusion \& mesial movement of lower molars within both groups were found. However no significant difference was found when the Begg mechano-therapy was compared to PEA Technique on vertical dimensional changes.
\end{abstract}

\section{Introduction}

Extraction of teeth has always been the primary method of gaining space for either correction of severe crowding or retraction of proclined teeth. It also helps in closing the bite in open bite subjects and has therefore been suggested as a method of controlling the vertical dimension of the face. The vertical dimension of the face increased following orthodontic treatment as the molars were extruded because of inter-arch mechanics, which consequently caused clockwise rotation of the mandible. Further fuelling this controversy, Cusimano and McLaughlin (1993) and Upadhyay et al. (2008) suggested that occlusal movement of the posterior teeth tends to keep pace with the increase in anterior face height, thus maintaining the mandibular plane angle and nullifying any bite-closing effect of protraction of the posterior teeth.

Swain and Ackerman (1969) and Williams (1977) reported considerable molar extrusion under the influence of Class II elastics. However, James (1968) and Thompson (1972) found that with the Begg appliance, the deep bite was corrected primarily by simultaneous extrusion of the mandibular molars and intrusion of the lower incisors. It was suggested that molar extrusion might lead to a backward rotation of the mandible increasing the vertical facial dimension with a potentially detrimental effect on facial aesthetics.

The aim of this research was to evaluate and compare the changes in the vertical facial dimension in Angle Class I bimaxillary dentoalveolar protrusion subjects on an underlying Class I or mild Class II skeletal base treated with first four premolar extractions with the Begg or PEA(MBT) technique.

\section{Subjects and methods}

The study design was retrospective in nature and the sample was randomly collected from the orthodontic records at the Department of Orthodontics, CSMSS Dental college \& Hospital, Aurangabad, Maharashtra, India. No specific criteria were set for prescribing the appliances and the patients were arbitrarily divided into two groups-Begg's and PEA.

The initial and final records of all patients who initially presented with an Angle Class I bimaxillary dentoalveolar protrusion and were treated with the Begg or PEA technique were obtained and every alternate patient from both groups was selected.

The inclusion criteria were an Angle Class I bimaxillary dentoalveolar protrusion on an underlying Class I or mild Class II skeletal base. All permanent maxillary and mandibular teeth were present. Proclined upper and lower incisors as depicted by their pre-treatment cephalometric values. All patients had undergone therapeutic extraction of the four first premolars. No headgear or second molar banding or any other anchoragereinforcing appliance was used.

The exclusion criteria were incomplete pre- or post-treatment records, congenitally missing teeth (except third molars) or mutilated dentitions, periodontally affected cases where retraction of the teeth could be compromised, and those with congenital anomalies or significant facial asymmetry.

The final sample comprised 40 Class I bi-maxillary protrusive patients: Begg appliance group $(N=20)$ with a mean age of $18-20$ years and PEA group $(N=20)$ with a mean age of $18-20$ years. 


\section{Treatment protocol}

Begg mechanotherapy.

All teeth were bonded and the first molars were banded. Following initial alignment, stage I (bite opening) was carried on 0.016 inch Australian stainless steel archwire (A.J. Wilcock) with Class II elastics. After achieving an edge-to-edge bite, stage II (space closure) was performed on a 0.018 inch Australian stainless steel archwire with Class I and II elastics. Stage III (torquing and root uprighting) was performed on a 0.020 inch premium Australian stainless steel base archwire with 0.014 inch premium plus Australian stainless steel torquing auxiliary, uprighting springs, and Class II elastics.

\section{MBT mechanotherapy:}

For the MBT group, 0.022 inch slot MBT prescription was used. After initial levelling and alignment, enmass retraction was carried out using an elastic chain on 0.018 inch stainless steel archwire Bite opening, if necessary, was undertaken with a $0.017 \times 0.025$ inch stainless steel intrusion arch either in the upper or lower arch.

\section{Cephalometric analysis: ( Figure no.1)}

Cephalometric radiographs at the beginning (T1) and end (T2) of treatment were selected. Since the T1 and T2 cephalograms were taken on the same machine, the magnification of the cephalograms was not considered. The lateral films were hand traced by one investigator (AC) under the same illumination and magnification on a single matte lacquered polyester acetate tracing paper of using a $3 \mathrm{H}$ lead pencil. Structures appearing as bilateral images were identified by bisecting the outlines of the images. The 13 parameters studied were broadly divided into skeletal and dental parameters. The skeletal parameters were further divided into linear \& angular measurements.

The skeletal linear measurements were total face height (N-Me), lower anterior face height (LAFH), posterior face height $(\mathrm{PFH})$, face height ratio (N-ANS/ANS-Me), and Jarabak's ratio (PFH:AFH). The skeletal angular measurements were FMA, SN-GoGn, and the $\mathrm{Y}$ axis. The dental parameters were lower molar to mandibular plane (LM-MP), lower molar to PTM perpendicular $(\mathrm{LM}-\mathrm{PTM} \perp)$, upper molar to palatal plane (UM-PP), and upper molar to PTM perpendicular

$(\mathrm{UM}-\mathrm{PTM} \perp)$, upper incisor edge to palatal plane.

Face height - Linear measurement from nasion $(\mathrm{N})$ to menton $(\mathrm{Me})$

Lower anterior face height - Linear measurement from anterior nasal spine (ANS) to Me.

Posterior face height - Linear measurement from sella (S) to gonion (Go)

Face height ratio - The ratio between upper face height and lower anterior face height (N-ANS/ANS-Me)

Jarabak's ratio - The ratio between posterior and anterior face height. (S-Go/N-Me)

FMA - The angle formed between the FH plane and mandibular plane (Go-Me)

SN-GoGn - The angle formed between the SN line and mandibular plane (Go-Gn)

Y axis - The angle between $\mathrm{S}-\mathrm{Gn}$ line to the FH plane.

Lower molar to mandibular plane - Perpendicular distance from the mandibular plane (Go-Me) to the central fossa of the mandibular first permanent molar

Lower molar to PTM perpendicular - Linear measurement on a perpendicular line drawn from PTM point to the central fossa of the mandibular first permanent molar

Upper molar to palatal plane - Perpendicular distance from the palatal plane (ANS-PNS) to the central fossa of the maxillary first permanent molar

Upper molar to PTM perpendicular - Linear measurement on a perpendicular line drawn from PTM point to the central fossa of the maxillary first permanent molar

Upper Incisor to palatal plane - Linear measurement on palatal palne drawn from upper central incisal edge 


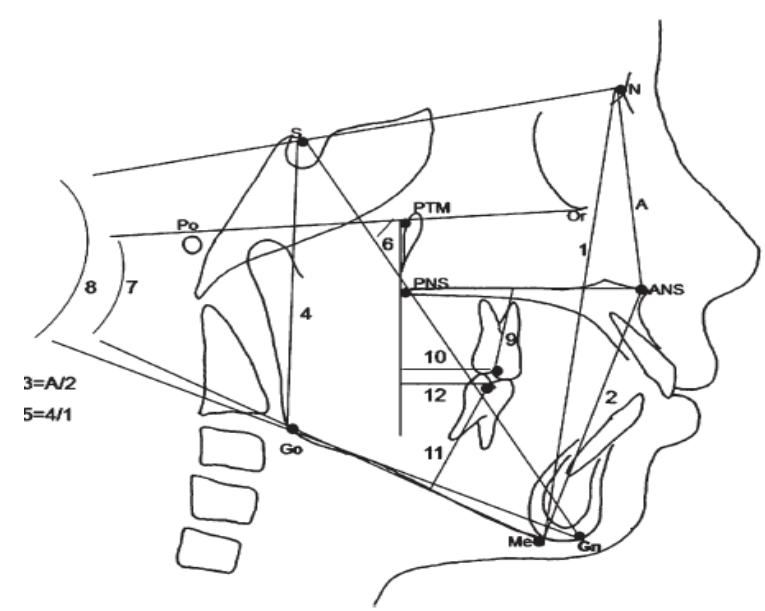

\section{Statistical analysis}

Fig No.1

All analyses were performed using the Statistical Package Minitab15 english was used. The mean and standard deviation for each cephalometric variable were determined. The following parametric statistical tests were used: two tailed paired $t$-tests (to determine the significance of changes in the groups after treatment) and unpaired $t$-tests (to determine the differences between the two groups either pre- or post-treatment). A confidence level greater than 5 per cent $(P>0.05)$ was not considered significant.

TABLE NO.1

\begin{tabular}{|c|c|c|c|c|}
\hline \multirow{2}{*}{} & \multicolumn{2}{|c|}{ PEEG } \\
\cline { 2 - 5 } & mean & SD & Mean & SD \\
\hline $\begin{array}{c}\text { Skeletal linear } \\
\text { measurements }\end{array}$ & & & & \\
\hline TFH & 109.7 & 5.23 & 112.50 & 4.84 \\
\hline LAFH & 63.4 & 3.062 & 66.30 & 3.093 \\
\hline PFH & 71.50 & 3.63 & 73.70 & 3.40 \\
\hline UFH/LAFH & 0.744 & 0.05 & 0.712 & 0.048 \\
\hline PFH/TFH & 0.650 & 0.026 & 0.652 & 0.025 \\
\hline $\begin{array}{c}\text { Skeletal Angular } \\
\text { Measurements }\end{array}$ & & & & \\
\hline FMA & 32 & 4.81 & 32.90 & 5.30 \\
\hline SnGoGn & 28.70 & 2.359 & 29.20 & 1.874 \\
\hline Y axis & 63.50 & 3.028 & 64.50 & 2.79 \\
\hline Uental Measurements & & & & \\
\hline LM-MP(mm) & 28.50 & 2.79 & 30.20 & 2.93 \\
\hline LM-PTMt(mm) & 25.80 & 2.78 & 25.70 & 2.983 \\
\hline UM-PP(mm) & 22.20 & 1.75 & 22.40 & 1.578 \\
\hline UM-PTMt (mm) & 23.30 & 2.791 & 24.10 & 1.524 \\
\hline UI-PP(mm) & 33.20 & 2.394 & 30.70 & 1.33 \\
\hline
\end{tabular}

TABLE NO. 2

\begin{tabular}{|c|c|c|c|c|}
\hline \multirow{2}{*}{} & \multicolumn{2}{|c|}{ PEA(MBT) } & \multicolumn{2}{c|}{ Post Treatment } \\
\cline { 2 - 5 } & mean & SD & Mean & \\
\hline $\begin{array}{c}\text { Skeletal linear } \\
\text { measurements }\end{array}$ & & & & 5.14 \\
\hline TFH & 112.8 & 4.66 & 115.00 & 5.25 \\
\hline LAFH & 64.4 & 5.48 & 66.40 & 3.06 \\
\hline PFH & 76.10 & 3.31 & 77.70 & 0.0683 \\
\hline UFH/LAFH & 0.803 & 0.093 & 0.763 & 0.173 \\
\hline PFH/TFH & 0.691 & 0.026 & 0.748 & \\
\hline $\begin{array}{c}\text { Skeletal Angular } \\
\text { Measurements }\end{array}$ & & & & \\
\hline
\end{tabular}




\begin{tabular}{|c|c|c|c|c|}
\hline FMA & 25.70 & 4.35 & 26.60 & 4.81 \\
\hline SnGoGn & 26.80 & 3.61 & 28.30 & 4.64 \\
\hline Y axis & 61.50 & 5.64 & 62.00 & 4.74 \\
\hline Dental Measurements & & & & \\
\hline LM-MP(mm) & 28.60 & 2.72 & 30.60 & 3.66 \\
\hline LM-PTM-(mm) & 25.30 & 3.59 & 27.40 & 3.24 \\
\hline UM-PP(mm) & 21.00 & 3.018 & 21.7 & 2.627 \\
\hline UM-PTM† $(\mathrm{mm})$ & 22.20 & 3.29 & 23.390 & 3.07 \\
\hline UI-PP(mm) & 27.20 & 4.64 & 27.30 & 4.00 \\
\hline
\end{tabular}

TABLE NO.3

\begin{tabular}{|c|c|c|c|c|c|c|}
\hline & \multicolumn{2}{|c|}{ BEGG } & \multicolumn{2}{|c|}{ PEA(MBT) } & \multirow[b]{2}{*}{$\mathrm{P}$ value } & \multirow[b]{2}{*}{ Significance } \\
\hline & mean & $\mathrm{SD}$ & Mean & SD & & \\
\hline \multicolumn{7}{|c|}{ Skeletal linear measurements } \\
\hline TFH & 3 & 1.49 & 2.60 & 1.07 & 0.501 & NS \\
\hline LAFH & 2.90 & 1.20 & 2 & 1.33 & 0.131 & NS \\
\hline PFH & 2.20 & 0.91 & 1.8 & 1.23 & 0.422 & NS \\
\hline UFH/LAFH & -0.032 & 0.014 & 0.048 & 0.030 & 0.161 & NS \\
\hline PFH/TFH & 0.019 & 0.012 & 0.077 & 0.17 & 0.311 & NS \\
\hline \multicolumn{7}{|l|}{$\begin{array}{c}\text { Skeletal Angular } \\
\text { Measurements }\end{array}$} \\
\hline FMA & 2.30 & 1.49 & 1.50 & 1.18 & 0.201 & NS \\
\hline SnGoGn & 1.70 & 0.675 & 0.210 & 1.37 & 0.423 & NS \\
\hline Y axis & 1.6 & 1.17 & 1.3 & 0.823 & 0.518 & NS \\
\hline \multicolumn{7}{|l|}{ Dental Measurements } \\
\hline LM-MP(mm) & 1.70 & 1.25 & 2 & 1.63 & 0.651 & NS \\
\hline LM-PTMH(mm) & -1.73 & -1.10 & 2.09 & 0.831 & 0.394 & NS \\
\hline UM-PP(mm) & 1.27 & 1.01 & 1.54 & 0.82 & 0.495 & NS \\
\hline UM-PTM† (mm) & 2 & 1.95 & 1.91 & 1.14 & 0.895 & NS \\
\hline $\mathrm{UI}-\mathrm{PP}(\mathrm{mm})$ & -2.27 & -1.95 & 2.27 & 1.27 & 1 & NS \\
\hline
\end{tabular}

NS, not significant. $* \mathrm{P}<0.05 ; * * \mathrm{P}<0.01, * * * \mathrm{P}<0.001$

\section{Results}

Cephalometric measurements of the two groups at $\mathrm{T} 1$ and $\mathrm{T} 2$ were calculated.

Changes in the Begg group_(TABLE no.1)

The Begg group showed a significant increase for the skeletal linear measurements of TFH $(3 \pm 1.49 \mathrm{~mm})$; LAFH $(2.9 \pm 1.2 \mathrm{~mm})$; PFH $(2.2 \pm 0.9 \mathrm{~mm})$ and facial height ratio $(-0.032 \pm 0.014 \mathrm{~mm}$;), while no significant changes were observed for Jarabak's ratio $(0.002 \pm 0.012 \mathrm{~mm})$.

In addition, no significant differences were recorded in the skeletal angular measurements for $\mathrm{Y}$ axis $(1.6 \pm 1.17)$, FMA $(2.30 \pm 1.49)$, and $\mathrm{Sn}-\mathrm{GoGn}(1.7 \pm 0.065)$. However, for the dental parameters, significant changes were observed for LM-MP $(1.7 \pm 1.25 \mathrm{~mm})$, and UI-PP $(-2.5 \pm 1.95 \mathrm{~mm})$. UM-PP $(1.27 \pm 1.01 \mathrm{~mm})$, UM-PTM $(2 \pm 1.9 \mathrm{~mm}), \operatorname{LM}-\mathrm{PTM}(-0.1 \pm 2 \mathrm{~mm})$ did not show any significant differences between $\mathrm{T} 2$ and T1 values.

Changes in the PEA group_(TABLE no. 2)

The PEA group had a highly significant increase in skeletal linear measurements of TFH $(2.6 \pm 1.07$ $\mathrm{mm})$; and LAFH $(2 \pm 1.33 \mathrm{~mm}) ; P<0.01)$ PFH $(1.8 \pm 1.23 \mathrm{~mm}$; $)$ and facial height ratio $(-0.048 \pm 0.03 \mathrm{~mm}$; $)$, while no significant changes were observed for Jarabak's ratio $(0.077 \pm 0.17)$.

No significant differences were observed for skeletal angular measurements: $\mathrm{Y}$ axis $(1.3 \pm 0.82)$, FMA $(1.51 \pm 1.18)$, except $\mathrm{Sn}-\mathrm{GoGn}(2.1 \pm 1.37)$. Dental measurements showed highly significant changes for LMPTM $(2.1 \pm 0.83 \mathrm{~mm}) \&$ UM-PTM $\perp(1.71 \pm 1.14 \mathrm{~mm}), \mathrm{LM}-\mathrm{MP}(2 \pm 1.63 \mathrm{~mm})$ and no significant changes were observed with UM-PP $(1.54 \pm 0.82 \mathrm{~mm}) \&$ UI-PP $(2.27 \pm 1.27 \mathrm{~mm})$.

\section{Comparison of Begg and PEA groups (Table No.3)} parameters.

No significant differences were found between the groups for any skeletal (linear and angular) or dental 


\section{Discussion}

This retrospective study cephalometrically compare the treatment effects of the conventional Begg technique with the PEA(MBT) in terms of their effect on the vertical facial dimensions.

Statistically significant intra-group differences were observed for both techniques for TFH, LAFH, PFH \& facial height ratio and vertical of lower molars and upper incisor, mesial movement of upper and lower molars with the exception of UM-PTM $\perp$, LM-PTM for the Begg group. However, none of the parameters showed any statistically significant difference when the Begg group was compared with the PEA group.

TFH increased by $3 \mathrm{~mm}$ in the Begg group and $2.6 \mathrm{~mm}$ in the PEA group which could be attributed to the extrusion of the molars with both techniques and is in accordance with the findings of Ahn and Schneider (2000) and Kim and Kim (2005).

LAFH showed an increase of $2.90 \mathrm{~mm}$ for the Begg group as compared with $2 \mathrm{~mm}$ for the PEA group, which could be attributed to molar extrusion. In the Begg group, this increase could be attributed to the use of Class II elastics having a vertical component of force causing the molar extrusion. The increase in face height was similar to the observations of James (1968), Sarisoy and Darendeliler (1999), Ahn and Schneider (2000), and $\mathrm{Kim}$ and $\mathrm{Kim}$ (2005).

The increase in the PFH was similar for the two groups. In the Begg group, both PFH and LAFH increased by almost the same amount thereby indicating a possible forward translation of the mandible under the Class II elastic traction force similar to that reported by Payne (1971) and Begg and Kesling (1977) On the other hand, in the PEA group, LAFH increased marginally more than PFH indicating a very mild opening of the mandibular plane angle.

Face height ratio decreased in both groups due to the increase in LAFH; it reduced more in the PEA group because of the greater increase in LAFH than in the Begg group. The value was similar to that observed by Staggers (1994). Their difference was however insignificant.

A similar result was obtained for Jarabak's ratio for both groups indicating good maintenance of the vertical dimension with both techniques. Inspite of both upper and lower molar extrusion, the mandibular plane angle remained the same in the Begg group and had a minimal clockwise rotation in the PEA group probably indicating that mesial movement of the molars compensated for their extrusion thus maintaining the vertical facial dimensions.

The $Y$ axis increased in the Begg sample by only 1.6 degrees indicating a very mild opening of the mandibular plane angle. similarly, in the PEA group, again though statistically insignificant, the $Y$ axis increased by 1.3 degrees indicating a mild opening of the mandibular plane angle similar to the observations of Ahn and Schneider (2000) and Kim and Kim (2005).

FMA and $\mathrm{Sn}-\mathrm{GoGn}$ in the Begg group though indicated excellent control of the vertical dimension slight amount of opening of the mandibular plane angle, whereas in the PEA group, both parameters showed a marginal increase indicating a non-significant clockwise rotation of the mandible similar to the observations of Gianelly (1984), Kocadereli (1999), and Kim and Kim (2005) but contrary to the findings of Garlington and logan (1990) and Hayasaki and Henriques (2005) who reported a 0.8 degree reduction in the mandibular plane angle. An increase in the mandibular plane angle was also reported by Kottraba (1971), Dougherty (1968).

The upper molar to palatal plane angle showed a non-significant extrusion of the upper molar with the Begg technique of $1.27 \mathrm{~mm}$ as compared with $1.54 \mathrm{~mm}$ with the PEA. The lower molar to palatal plane showed a significant extrusion of $1.7 \mathrm{~mm}$ with the Begg technique and $2 \mathrm{~mm}$ with the PEA, indicating that both systems were similar in the extrusion of the molars.

The extrusion of the lower molars in the Begg group was caused primarily by the vertical component of the force of the Class II elastics and the anchor bends which tend to tip and extrude the molars (Campe et al., 1967; James, 1968; Bijlstra, 1969; McDowell, 1969; Thompson, 1979).

In the PEA group, this extrusion could probably be attributed to the bite opening mechanics employed (Ahn and Schneider, 2000; Hayasaki and Henriques, 2005).

The upper and lower molars to PTM perpendicular showed that the upper molar migrated $2 \mathrm{~mm}$ mesially in the Begg group and $1.91 \mathrm{~mm}$ in the PEA group. The lower molar also migrated $1.2 \mathrm{~mm}$ in the Begg group and $2.1 \mathrm{~mm}$ in the PEA group showing that the Begg technique was marginally better at conserving anchorage than the PEA. In the Begg group, the anchorage loss could be attributed to the high reactionary forces exerted during torquing of the anterior teeth.

While the current study was performed pre- and post treatment, long-term evaluation is advocated to obtain a clearer picture of retention of the vertical dimensional changes.

\section{Conclusion}


1. None of the observed parameters showed any statistically significant difference when the Begg and the PEA group were compared.

2. In the intra-group comparisons, a statistically significant difference was observed with both techniques for

TFH, LAFH, PFH, and mesial movement and extrusion of both the upper and lower molars.

3. There was no significant difference when the Begg mechano-therapy was compared with the PEA technique at T2 for evaluation of vertical dimensional changes, contrary to the general consensus that Begg mechanotherapy is associated with a greater increase in vertical facial dimensions.

4. According to above study we can conclude that choice of treatment mechanics whether to use Beggs mechano-therapy or PEA mechano-therapy is independent of the growth pattern.

\section{References}

[1]. Ahn J G, Schneider B J 2000 Cephalometric appraisal of post-treatment vertical changes in adult orthodontic patients. American Journal of Orthodontics and Dentofacial Orthopedics 118: 378-384.

[2]. Barton J J 1973 A cephalometric comparison of cases treated with edgewise and Begg techniques. Angle Orthodontist 43: 119-146

[3]. Bijlstra R 1969 Vertical changes during Begg technique. Transactions of the European Orthodontic Society pp. 385-396

[4]. Chua A L 1993 The effects of extraction versus nonextraction orthodontic treatment on the growth of the lower anterior face height. American Journal of Orthodontics and Dentofacial Orthopedics 104: 361-368.

[5]. Cusimano C, McLaughlin R P 1993 Effects of first bicuspid extractions on facial height in high-angle cases. Journal of Clinical Orthodontics 27:594-598

[6]. Fischer J C 1974 A cephalometric comparison of vertical dimension changes in Begg v/s Edgewise cases during treatment. Thesis, St. Louis University

[7]. Hans M G, Groisser G 2006 Cephalometric changes in overbite and vertical facial height after removal of 4 first molars or first premolars.American Journal of Orthodontics and Dentofacial Orthopedics 130:183-188

[8]. Isaacson J R 1971 Extreme variation in vertical facial growth and associated variation in skeletal and dental relations. Angle Orthodontist 41: 219-229

[9]. James T A 1968 Changes in vertical relationships of teeth during and following use of the Begg light wire differential. American Journal of Orthodontics 54: 152

[10]. Kashani M A, Neishabori A 2003 The effect of quadrilateral first premolar extractions on vertical occlusal dimensions. European Journal of Orthodontics 25: 530-531

[11]. Kim T K, Kim J T 2005 First or second premolar extraction effects on facial vertical dimension. Angle Orthodontist 75: 177-182

[12]. Kocadereli I 1999 The effect of first premolar extraction on vertical dimension. American Journal of Orthodontics and Dentofacial Orthopedics 116: 41-45

[13]. Pearson L E 1973 Vertical control through use of mandibular posterior intrusive forces. Angle Orthodontist 43: 194-200

[14]. Pearson L E 1978 Vertical control in treatment of patients having backward rotational growth tendencies. Angle Orthodontist 48:132-140

[15]. Staggers J A 1994 Vertical changes following first premolar extractions. American Journal of Orthodontics and Dentofacial Orthopedics 105: 19-24

[16]. Thompson W J 1979 Occlusal plane and overbite. Angle Orthodontist 49:47-55

[17]. Tulley W J 1959 Role of extraction in orthodontic treatment. British Dental Journal 107: 199-205. 